\title{
DIVERSIDAD GENÉTICA DE ROSA DE JAMAICA EN GUATEMALA REVELADA POR MARCADORES AFLP ${ }^{1}$
}

\author{
Karla Melina Ponciano-Samayoa ${ }^{2}$,Sergio Gonzalo Hidalgo-Villatoro ${ }^{2}$
}

\section{RESUMEN}

Diversidad genética de rosa de jamaica en Guatemala revelada por marcadores AFLP. El objetivo de este estudio fue caracterizar la diversidad genética existente en una colección de diecisiete genotipos de rosa de jamaica cultivados en Guatemala. Durante el período julio 2010/mayo2011, en el Instituto de Ciencia y Tecnología Agrícolas (ICTA) se amplificaron doce combinaciones selectivas AFLP de las cuales cinco fueron altamente informativas. Se observó un 98\% de loci polimórficos, con un promedio de nueve por amplificación selectiva. Se estableció que no hay genotipos duplicados en la colección. El análisis de agrupamientos y de correspondencia identificó tres grupos muy cercanos entre sí. El cuarto grupo fue conformado por la accesión 205 que se caracteriza por ser muy precoz y tener alto rendimiento respecto al resto. Los agrupamientos formados coincidieron con los conglomerados identificados en la caracterización agromorfológica realizada a la colección según su precocidad, días a cosecha y rendimiento. Es probable que exista un ligamiento entre AFLPs y loci de características cuantitativas, que puede ser aprovechado en selección asistida por marcadores. La diversidad genética (Nei) fue alta $(0,3053)$ de la cual el 57,62\% se debió a la diferenciación entre grupos $\left(\mathrm{G}_{\mathrm{ST}}\right)$. La variación genética dentro de los grupos fue menor $(42,38 \%)$ y probablemente se debe al bajo flujo genético $(\mathrm{Nm}=0,3678)$ que hay en una especie autógama.

\begin{abstract}
Genetic diversity of roselle in Guatemala revealed by AFLP markers. The objective of this study was to characterize a collection of 17 genotypes of roselle that were collected in Guatemala. During July 2010/ May 2011, twelve selective combinations of AFLP were amplified at Instituto de Ciencia y Tecnología Agrícolas (ICTA), to identify clusters and to analyze the genetic diversity within the collection. A $98 \%$ of polymorphic loci was observed, nine per amplification in average. Five selective primer pairs were high informative and are recommended for future investigations. No duplicated genotypes were identified. The clustering and correspondence analysis identified three closely related groups. A fourth group included accession 205, characterized by early maturity and high yield. The clusters formed were similar to those identified in a previous agromorphological characterization. Probably, there is a genetic linkage between AFLPs and quantitative trait loci (QTL ) that can be used in marker-assisted selection. The genetic diversity (Nei) was high (0.3053). The genetic differentiation $\left(\mathrm{G}_{\mathrm{ST}}\right)$ between groups was $57.62 \%$. The genetic variability within clusters was lower $(42.38 \%)$ and probably due to the low genetic flow $(\mathrm{Nm}=0.3678)$ commonly found in inbreed species.
\end{abstract}

Key words: Hibiscus sabdariffa L., similarity, clustering, genetic differentiation, genetic flow.

Palabras clave: Hibiscus sabdariffa L., similaridad, agrupamientos, diferenciación genética, flujo genético.

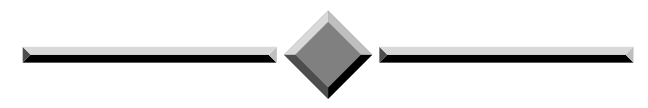

\footnotetext{
1 Recibido: 28 de junio, 2011. Aceptado: 12 de marzo, 2012. Proyecto de investigación del Laboratorio de Biotecnología y el Programa de Frutales. Instituto de Ciencia y Tecnología Agrícolas ICTA, Km. 21,5 Carretera al Pacífico, Bárcenas, Villa Nueva, Guatemala, C.A.

2 Laboratorio de Biotecnología y Programa de Frutales. Instituto de Ciencia y Tecnología Agrícolas ICTA. Km. 21.5 Carretera al Pacífico, Bárcenas, Villa Nueva, Guatemala, C.A. kponciano@hotmail.com; sergiohidalgo_1@hotmail.com
} 


\section{INTRODUCCIÓN}

La rosa de jamaica (Hibiscus sabdariffa L.) es una planta dicotiledónea de la familia Malvaceae originaria de África (Gómez et al. 2008). Actualmente, está adaptada a las zonas tropicales de Asia, Oceanía y América (Gómez et al. 2008). Se considera una especie predominantemente autógama diploide $(2 n=72)$ (Alcocés 2009). También es conocida como flor de Jamaica, rosa de Jericó, karkade y roselle (Solórzano y Macario 2002).

La planta crece como un arbusto anual y algunas veces bianual, tienen tallo semileñoso, con raíz pivotante, hojas de color verde alternas de $8,89 \mathrm{~cm}$ de largo. Las flores son axilares y solitarias. La corola es acampanada de color amarillo pálido o rosado con un círculo de coloración púrpura en la parte interna. Al marchitarse, desaparece y deja el cáliz libre. Este cáliz se mantiene y alarga mientras se torna carnoso de un color rojo obscuro. El fruto tiene una forma capsular que contiene al menos 20 semillas de color negro (Hidalgo et al. 2009).

El cáliz es la parte más apreciada de la planta. Se usa ampliamente para producir bebidas o infusiones (té), vinos, compotas, postres, salsas, dulces, pasteles, uso culinario y coloración de embutidos (Rojas 1999). Debido a su alto contenido de antocianinas y ácidos hibiscus, los cálices tienen un gusto ácido dando un gusto particular a los productos preparados con ellos. Sus propiedades hacen que sea utilizada en medicina natural como un regulador de la presión arterial, de la función hepática y como tratamiento de la pirexia; además se conoce como antimicrobiano, diurético, digestivo y sedativo. Es muy conocida por su acción antioxidante (Gómez et al. 2008).

Es un cultivo susceptible al ataque de patógenos como Phytophtora parasitica, Phoma sabdariffae, Macrophomina phaseolina, Phizoctonia solani, Botrytis cinerea, Sclerotium rolfsii y Conella musaiaensis var. hibisci. El rendimiento está entre 0,5 a 2 toneladas de cáliz por hectárea según la variedad (Gómez et al. 2008).

En Guatemala, H. sabdariffa L. se siembra en pequeña escala en los departamentos de Baja Verapaz, Huehuetenango y algunas áreas de las costas del Pacífico. Se encuentra en zonas que tienen altitudes de $0-1200 \mathrm{msnm}$, temperaturas entre 22 y $30^{\circ} \mathrm{C}$ y precipitación pluvial de 800 a 2000mm al año. En la actualidad, se considera un cultivo subutilizado de potencial económico importante pero que se tiene relegado a siembras temporales en suelos marginales con poca fertilidad (Hidalgo et al. 2009).

Hidalgo et al. (2009) realizaron una colecta de genotipos de $H$. sabdariffa en las zonas productoras de Guatemala con la que se inició una pequeña colección de trece accesiones que fue utilizada para establecer un programa de mejoramiento genético con resultados excelentes. La liberación de la variedad mejorada ROSICTA que beneficia a los agricultores guatemaltecos que están exportando su producción.

La caracterización agromorfológica de la colección constituida se hizo a partir de 24 caracteres cuantitativos y siete cualitativos. Las accesiones se agruparon de acuerdo a su ciclo de crecimiento y cosecha en tardíos, intermedios y precoces. Tres accesiones, identificadas como 0205, 1205 y 1305, se catalogaron como promisorias debido a su precocidad y rendimiento sobresaliente. En las recomendaciones se estableció que combinar ciclos de cosecha permite maximizar los recursos y diversificar las fincas productoras (Hidalgo et al. 2009).

A pesar de la importancia de H. sabdariffa L., poco se conoce de su estructura genética. Sin embargo, por los resultados observados en campo, se sospecha que tiene una amplia diversidad genética como resultado de la distribución genética y adaptación en sitios de introducción como Guatemala. Pocos autores han reportado sus esfuerzos en el establecimiento de la diversidad genética de la rosa de Jamaica utilizando la técnica de RAPDs en países como Egipto, Estados Unidos y México (Hussein et al. 2010, Jendereck et al. 1997, Gómez et al. 2008). Menos frecuentes son los estudios hechos con microsatélites (Torres et al. 2011) y AFLP. La técnica de AFLP genera una huella de ADN, dentro de un sistema ideal de marcadores para revelar la diversidad genética presente entre individuos, poblaciones y especies. Sus características la hacen una técnica altamente reproducible, con alta cobertura del genoma y sin requerir de una secuencia de ADN previa. Esta última característica hace que puedan amplificarse marcadores en especies que no han sido muy estudiadas y que toda la información generada sirva de base para la construcción de mapas genéticos, estudios de genética de poblaciones y de interacción genotipo-ambiente (Tang et al. 2003). Ese fue el caso del estudio de diversidad genética en 
genotipos de Hibiscus tiliaceus L. (Tang et al. 2003) y Hibiscus cannabinus L. (Cheng et al. 2004) en China. Estos reportes de amplificación de AFLPs en especies del género Hibiscus fueron los más cercanos a $H$. sabdariffa L. No se encontró estudios a nivel mundial en esta especie.

El objetivo de esta investigación fue caracterizar la diversidad genética existente en una colección de diecisiete genotipos de $H$. sabdariffa cultivados en Guatemala con base en los patrones de amplificación con doce combinaciones selectivas de AFLPs para establecer similitudes y agrupamientos.

\section{MATERIALES Y MÉTODOS}

Material vegetal. En el período julio 2010/mayo 2011 se analizaron diecisiete accesiones de $H$. sabdariffa las cuales se colectaron y caracterizaron morfológicamente en un estudio anterior (Hidalgo et al. 2009). La identificación de los genotipos se muestra en el Cuadro 1.
Extracción, verificación de integridad y cuantificación de ADN. Las primeras hojas verdaderas de tres plántulas de dieciocho días de edad, se seleccionaron al azar para hacer una mezcla de material vegetal (aproximadamente $200 \mathrm{mg}$ ). La mezcla se maceró con pistilos y nitrógeno líquido dentro de tubos de microcentrífuga de 1,5 ul. El polvo obtenido se sometió al protocolo de extracción propuesto por Ponciano et al. 2009. El precipitado resultante se resuspendió en 150 $\mu l$ de buffer TE (10mM Tris-HCL, 1mM EDTA, pH $8,00)$ y se almacenó a $4^{\circ} \mathrm{C}$. La integridad del ADN se verificó cargando $10 \mu \mathrm{l}$ en un gel de agarosa al 0,8\%. A partir de diluciones 1:200 se cuantificó el ADN en un espectrofotómetro Bio-Rad SmartSpec3000 con un factor de conversión A260 nm 1,0=50,0 $\mu \mathrm{g} / \mathrm{ml}$. La calidad del ADN se determinó a partir de la razón A260/ A280. El ADN se diluyó a $25 \mathrm{ng} / \mu \mathrm{l}$ para las siguientes amplificaciones (Ponciano 2004).

Amplificación y visualización de AFLPs. Los recursos limitaron el estudio a la amplificación de doce combinaciones selectivas (Cuadro 2) que se seleccionaron con base en los resultados obtenidos en los pocos

Cuadro 1. Identificación de las accesiones de rosa de jamaica caracterizadas con AFLP. Guatemala, agosto 2009.

\begin{tabular}{cclc}
\hline$\#$ & Accesión ID & Municipio, Departamento de colecta & Altitud (msnm) \\
\hline 1 & 105 & Aldea Nueva Catarina, Jacaltenango, Huehuetenango & 730 \\
2 & 205 & Aldea El Limonar, Jacaltenango, Huehuetenango & 720 \\
3 & 305 & Aldea Pebil Pam, Jacaltenango, Huehuetenango & 720 \\
4 & 405 & Aldea La Laguna, Jacaltenango, Huehuetenango & 850 \\
5 & 505 & Aldea La Laguna, Jacaltenango, Huehuetenango & 720 \\
6 & 605 & Aldea La Laguna, Santa Rosa de Lima, Santa Rosa & 720 \\
7 & 705 & Caserío Nueva Lolita, San Andrés Villa Seca, Retalhuleu & 455 \\
8 & 805 & Aldea La Guitarra, Retalhuleu, Retalhuleu & 325 \\
9 & 905 & Rancho Pepe Km. 213, El Rosario, Champerico, Retalhuleu & 70 \\
10 & 1105 & Rancho Pepe Km. 213, El Rosario, Champerico, Retalhuleu & 70 \\
11 & 1205 & Nueva Libertad, Comalapa, Chiapas, México & 820 \\
12 & 1305 & Aldea Lochuyes, Santa Cruz El Chol, Baja Verapaz & 900 \\
13 & 1507 & UNAM, Naryt, México & 950 \\
14 & 1607 & Managua, Nicaragua & 242 \\
15 & 1707 & UNAM, Naryt, México & 950 \\
16 & 1807 & UNAM, Naryt, México & 950 \\
17 & 1907 & UNAM, Naryt, México & 950 \\
\hline
\end{tabular}


Cuadro 2. Amplificaciones selectivas utilizadas en la caracterización molecular de rosa de jamaica y número de polimorfismos amplificados. Guatemala, diciembre de 2010.

\begin{tabular}{ccc}
\hline Combinación AFLP & $\begin{array}{c}\text { No. bandas polimórficas } \\
\text { amplificadas }\end{array}$ \\
\hline 1 & E+ACA /M+CAA & 4 \\
2 & E+ACC /M+CAC & 6 \\
3 & E+ACG /M+CAG & 11 \\
4 & E+ACT /M+CAT & 9 \\
5 & E+ACA /M+CAC & 16 \\
6 & E+ACT /M+CAC & 17 \\
7 & E+AAG /M+CAG & 10 \\
8 & E+ACT /M+CAG & 2 \\
9 & E+AAG /M+CTC & 17 \\
10 & E+ACA /M+CTC & 9 \\
11 & E+ACT /M+CTC & 7 \\
12 & E+ACA /M+CTG & 11 \\
\hline
\end{tabular}

estudios similares generados con RAPDs (Hussein et al. 2010, Gómez et al. 2008) y al azar a modo de cubrir ampliamente las 64 posibilidades que permite la técnica. Para todas las muestras se amplificaron los marcadores con el sistema AFLP® Analysis System I (Invitrogen ${ }^{\mathrm{TM}}$ Life Technologies, California, USA) (Invitrogen Corporation 2003) siguiendo los protocolos establecidos por el proveedor. Se utilizó una alícuota de $10 \mu 1$ de ADN genómico total diluido en la reacción de digestión con las enzimas de restricción EcoR I y Mse I. Las reacciones se incubaron a $37^{\circ} \mathrm{C}$ por dos horas, seguido de una incubación a $70^{\circ} \mathrm{C}$ por quince minutos y una incubación en hielo por cinco minutos para desactivar las enzimas. Los fragmentos de ligación resultantes fueron ligados a adaptadores $E c o$ R I y Mse I para generar el ADN molde el cual fue utilizado en las amplificaciones siguientes. La preamplificación consistió en amplificar el ADN molde con partidores complementarios a los adaptadores y al sitio de restricción Mse I más un nucleótido adenina (Partidor Mse I $+\mathrm{A})$ y al sitio de restricción EcoR I más un nucleótido citosina (Partidor EcoR I +C). El programa de preamplificación consistió en 20 ciclos de desnaturalización a $94^{\circ} \mathrm{C}$ por 30 segundos, seguido de hibridización a $56^{\circ} \mathrm{C}$ por un minuto y una elongación a $72^{\circ} \mathrm{C}$ por un minuto.
La preamplificación de las muestras se verificó cargando $10 \mu \mathrm{l}$ de producto de PCR en un gel de agarosa $1,2 \%$. Las muestras se mezclaron con buffer de carga y corridas por treinta minutos a $50 \mathrm{~V}$ y se tiñeron con bromuro de etidio. En todas las muestras, el producto de preamplificación fue amplificado selectivamente utilizando combinaciones de partidores EcoR I y Mse I más tres nucleótidos selectivos adicionales cada uno (Cuadro 2). Estos marcadores se amplificaron por medio de PCR convencional en placas de 96 pozos en un termociclador ATC401 Apollo CLP. El programa de amplificación selectiva consistió en 36 ciclos distribuidos en dos etapas: Etapa 1. Trece ciclos de desnaturalización a $94^{\circ} \mathrm{C}$ por 30 segundos, seguido de hibridización a $65^{\circ} \mathrm{C}$ por 60 segundos y una elongación a $72^{\circ} \mathrm{C}$ por 60 segundos; con una reducción de $0,7^{\circ} \mathrm{C}$ en la temperatura de hibridización por cada ciclo sucesivo. Etapa 2. Veintitrés ciclos de desnaturalización a $94^{\circ} \mathrm{C}$ por 30 segundos, seguido de hibridización a $56^{\circ} \mathrm{C}$ por 60 segundos y elongación a $72^{\circ} \mathrm{C}$ por 60 segundos. Las mezclas de reacción finales se prepararon según las cantidades indicadas en el protocolo del proveedor (Invitrogen Corporation 2003).

Después de la amplificación, se agregó un volumen igual de solución de formamida al $95 \%$ y $3 \%$ agua, 2\% EDTA0.5M, 0,01\% azul de bromofenol y $0,01 \%$ xilen cianol. La mezcla se desnaturalizó por tres minutos a $94^{\circ} \mathrm{C}$ y durante dos minutos en hielo. Se realizó una electroforesis vertical en gel de poliacrilamida al 5\% (acrilamida:bis-acrilamida 29:1) en condiciones desnaturalizantes con $5 \mathrm{M}$ de urea en $1 \mathrm{X}$ TBE. Se corrieron en unidades de electroforesis BioRad Sequi-Gen GT a una potencia constante de 50W durante 1:15 horas. En todos los geles se incluyó una escalera de peso molecular de 100 pares de bases (pb). El procedimiento de tinción utilizado fue el descrito por Ponciano et al. (2009). El gel se secó por 24 horas y se obtuvo una imagen por scanner en formato JPEG y TIF.

Análisis de datos. A partir de los patrones de bandas visualizados en los geles se codificó la información alélica en una matriz de ausencia y presencia, la cual se utilizó para análisis de similaridad y de correspondencia en NTSYS-pc 2.02c (Rohlf 1992). Se utilizó el subprograma SIMQUAL para crear una matriz de distancias genéticas con coeficiente de Dice, la cual se utilizó en el subprograma SAHN para realizar un análisis de conglomerados y construir un 
dendrograma basado en el método de agrupamiento UPGMA y visualizarlos con el subprograma TREE PLOT. Con el subprograma CORRESP se construyó un diagrama en tres dimensiones mostrando la distribución de las accesiones en el espacio. La diversidad genética entre los grupos identificados en el dendrograma basada en distancias genéticas de Nei (Nei 1978) se calculó utilizando el programa POPGENE versión 1.32 (Yeh et al. 1997). La matriz utilizada para este programa se construyó según el formato de datos diploides dominantes. El análisis estadístico se realizó en los módulos DOMINANT DATA ANALYSISDIPLOID DATA- SINGLE POPULATIONS AND MULTIPLE POPULATIONS. Los parámetros de diversidad genética determinados fueron: número de loci polimórficos, porcentaje de loci polimórficos, índice de diferenciación genética $\left(\mathrm{G}_{\mathrm{ST}}\right)$, y flujo genético $(\mathrm{Nm})$. La diferenciación genética se calculó con la fórmula de Nei (1978) $\mathrm{G}_{\mathrm{ST}}=\mathrm{D}_{\mathrm{ST}} / \mathrm{H}_{\mathrm{T}}$ donde $\mathrm{H}_{\mathrm{T}}=\mathrm{H}_{\mathrm{S}}+\mathrm{D}_{\mathrm{ST}}$ y $\mathrm{D}_{\mathrm{ST}}=\mathrm{H}_{\mathrm{T}}-\mathrm{H}_{\mathrm{S}}$, donde $\mathrm{H}_{\mathrm{T}}$ es la diversidad total para las poblaciones, $\mathrm{H}_{\mathrm{S}}$ es la diversidad dentro de las poblaciones $\mathrm{y}_{\mathrm{ST}}$ es la diversidad genética entre poblaciones (Salvador et al. 1997, Blair et al. 2007). El flujo genético es igual al número de migrantes entre poblaciones por generación calculada en base a la fórmula $\mathrm{Nm}=0,5\left(1-\mathrm{G}_{\mathrm{ST}}\right) / \mathrm{G}_{\mathrm{ST}}$ (Blair et al. 2007). Otros parámetros generados de este mismo programa para cuantificar el grado de diferenciación entre y dentro de los grupos fueron: identidad genética (I), distancia genética de Nei (GD) y dendrograma UPGMA para representar las distancias genéticas entre grupos (Wagara et al. 2004).

\section{RESULTADOS Y DISCUSIÓN}

\section{Análisis de AFLPs en la colección de rosa de ja- maica}

Los patrones de amplificación de AFLPs de las accesiones de rosa de Jamaica (Figura 1) permitieron recopilar la información correspondiente a cada genotipo en 119 sitios polimórficos que fueron asignados como loci individuales debido a la naturaleza dominante de los AFLPs (Cuadro 2). En promedio, se observaron nueve polimorfismos por combinación selectiva. El rango fue de dos a veintisiete, donde la combinación menos informativa fue E+ACT / $\mathrm{M}+\mathrm{CAG}$. La combinación E+AAG/M+CAG reveló el número mayor de bandas polimórficas. Para estudios futuros, se deben utilizar las combinaciones $\mathrm{E}+\mathrm{ACG} / \mathrm{M}+\mathrm{CAG}, \mathrm{E}+\mathrm{ACA} / \mathrm{M}+\mathrm{CAC}, \mathrm{E}+\mathrm{ACT} /$ $\mathrm{M}+\mathrm{CAC}, \mathrm{E}+\mathrm{AAG} / \mathrm{M}+\mathrm{CTC}$ y $\mathrm{E}+\mathrm{ACA} / \mathrm{M}+\mathrm{CTG}$ por ser aquellas que amplificaron más de nueve loci polimórficos (Cuadro 2) como punto de partida y evaluar otras diferentes.

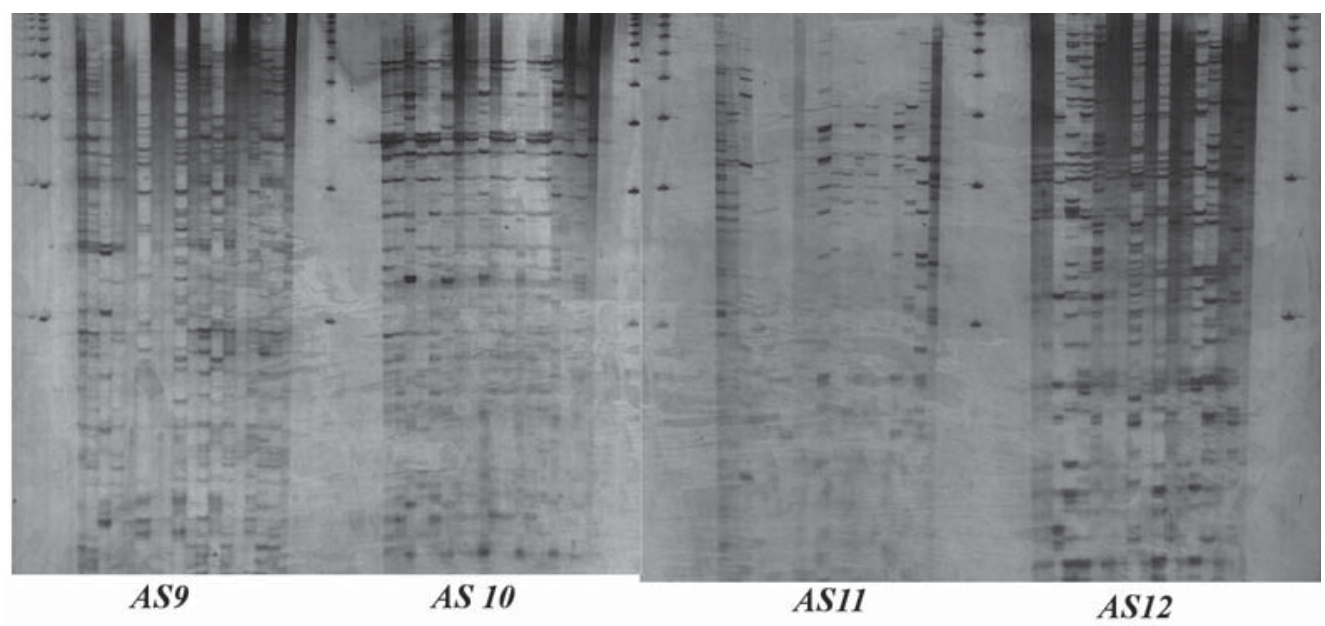

Figura 1. Patrones de bandas amplificados por las combinaciones selectivas 9, 10, 11, 12 para genotipos de rosa de jamaica visualizados en gel de poliacrilamida y tinción de plata. Guatemala, diciembre 2010. 


\section{Análisis de similaridad genética y agrupamientos}

Las relaciones genéticas entre los genotipos de rosa de Jamaica se establecieron a partir de una matriz de similaridad generada con el coeficiente de Dice (1945) que se representó en forma gráfica como dendrograma UPGMA (Figura 2). Se determinó que no existen genotipos duplicados. Esto coincide con lo reportado en la caracterización agromorfológica donde todos los genotipos fueron diferentes en sus características fenotípicas y de rendimiento (Hidalgo et al. 2009).

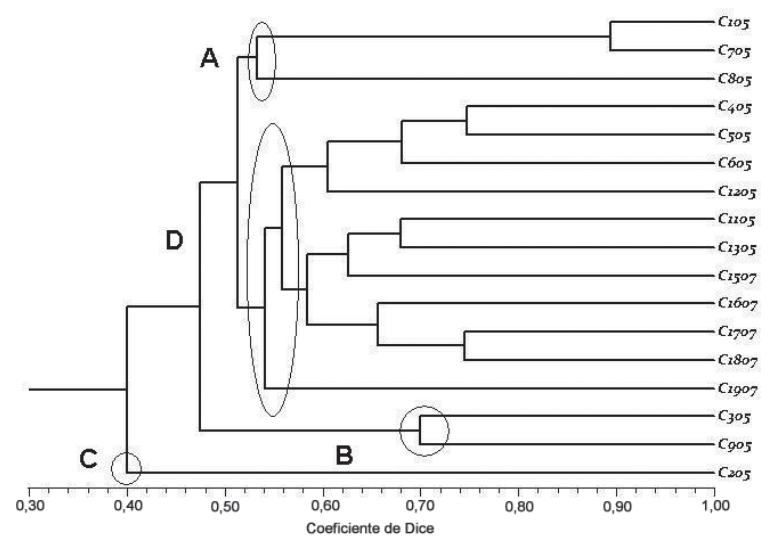

Figura 2. Dendrograma de la relación entre 17 genotipos de rosa de jamaica basada en el coeficiente de similaridad de Dice para doce combinaciones AFLP. Las letras A-D indican los grupos formados. Guatemala, mayo 2011.

Con una similaridad genética del $52 \%$ se conformaron tres agrupamientos (A, B y D). Se asignó como grupo C a la accesión 205, la cual se separó del resto con un coeficiente de similaridad de 0,40. En el grupo A, se reunieron los genotipos 105, 705 y 805 . De acuerdo a su ciclo de cosecha fueron clasificados como materiales tardíos (Hidalgo et al. 2009). El grupo D formó el conglomerado más grande con representantes de cosecha intermedia y tardía. Ahí se incluyó a los genotipos 405, 505, 605, 1205 en un subconglomerado; y a los genotipos 1105, 1305, 1505, 1605, 1705, 1805 y 1905, en otro. A diferencia de su relación agromorfológica, los genotipos 905 y 305 compartieron un coeficiente de similitud genética del $70 \%$ en el grupo B. Todos los materiales se agruparon por debajo de 0,90 de similaridad.
El análisis de correspondencia en dos dimensiones (Figura 3) hace evidente que las accesiones provenientes de México (1505, 1705, 1805, 1905) están emparentadas. El genotipo 1605, proveniente de Nicaragua, a pesar de ser diferente, tiene una mayor relación genética con los materiales mexicanos que con los guatemaltecos. También, el plano bidimensional acerca a los genotipos 905 y 305 con 405 y 505 . Esto resulta interesante, ya que el genotipo 305 fue clasificado como un material de cosecha tardía, pero puede ser que realmente sea intermedia, por lo que debió ser agrupado fenotípicamente con los materiales 405, 505 y 905. El genotipo 205 se observó fuera de las agrupaciones, lo que coincide con los resultados morfológicos donde este material mostró rendimientos sobresalientes y alta precocidad. Es posible que este material tenga un ancestro silvestre más lejano que le confiera de las diferencias genéticas necesarias para una mayor producción.

El material 1305 fue el segundo en precocidad y rendimiento (Hidalgo et al. 2009). Sin embargo, una vista en tres dimensiones de la correspondencia entre genotipos (Figura 4) muestra que dicho material guarda estrecha relación genética con los materiales de ciclo de cosecha intermedio. Es probable que su característica precoz analizada desde el punto de vista genético sea más un extremo de la variabilidad

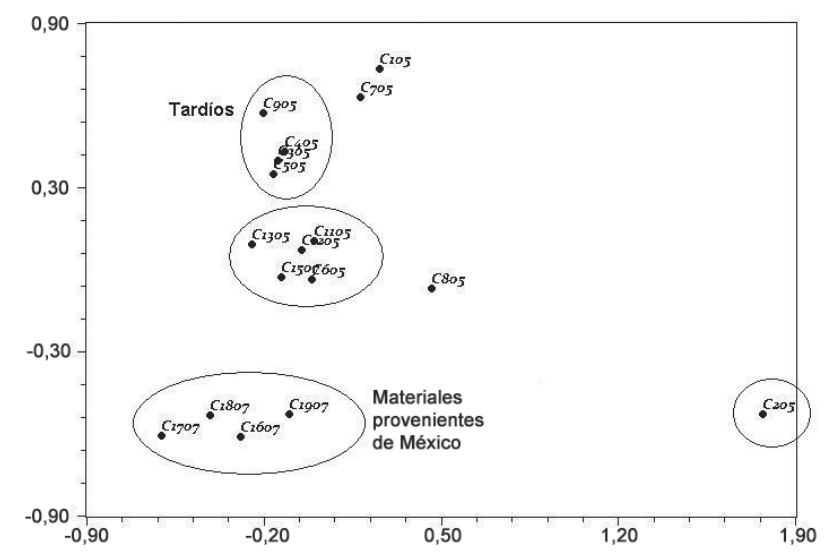

Figura 3. Análisis de correspondencia en dos dimensiones que muestra la relación entre 17 genotipos de rosa de jamaica con base en los datos de doce combinaciones AFLP. Guatemala, mayo 2011. 


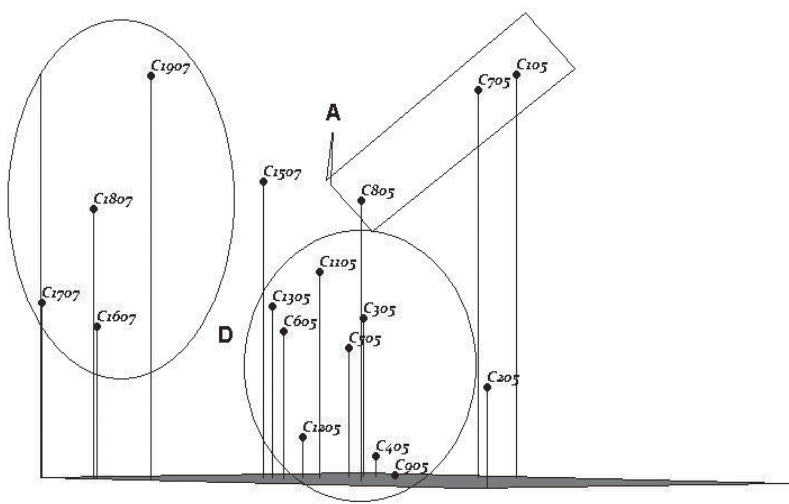

Figura 4. Análisis de correspondencia en tres dimensiones que muestra la relación entre 17 genotipos de rosa de jamaica con base en los datos de doce combinaciones AFLP. Guatemala, 2011.

encontrada entre los genotipos de cosecha intermedia que un material sobresaliente, como es el caso de la accesión 205.

La Figura 4 muestra la distribución tridimensional de los dos subgrupos del grupo D. El conglomerado de los genotipos 1607, 1707, 1807 y 1907 se agrupan cerca del eje $z$ mientras que otro conglomerado bien diferenciado incluye a las accesiones 1105, 1305 y 1507.

\section{Análisis de la diversidad genética}

La diversidad genética de Nei que representa a la colección completa fue 0,3053 . En los conglomerados formados, el valor de diversidad es proporcional al número de individuos (parámetro $\mathrm{N}$ ) incluidos en cada grupo (Cuadro 3). Por esta razón, el grupo D fue el de mayor valor y el grupo $\mathrm{C}$ no tiene valor. El porcentaje de loci polimórficos da una idea de la proporción de AFLP's que implican diferenciación entre los individuos de un agrupamiento.

La diversidad genética entre grupos en relación a la diversidad total fue 0,5762. Esto indica que el $57,62 \%$ de la diversidad total se debe a la diferenciación genética entre los grupos identificados. Mientras que la diferenciación de los genotipos dentro de cada grupo contribuye a un $42,38 \%$ al total. El flujo de genes fue bajo (menor que 1,0000$)(\mathrm{Nm}=0,3678)$ como se espera en plantas autógamas debido a que hay un limitado intercambio genético intra e interespecífico.

Los valores de identidad genética de los grupos identificados fueron altos y se encontraron en el rango de 0,5944 a 0,8679 (Cuadro 4). La representación gráfica de la relación genética entre grupos (Figura 5) indicó que $\mathrm{B}$ y $\mathrm{D}$ son los más similares $(\mathrm{I}=0,8679)$. Estos a la vez son cercanos al grupo $\mathrm{A}$. El grupo $\mathrm{C}$, conformado por el genotipo 205, es el de mayor distancia genética.

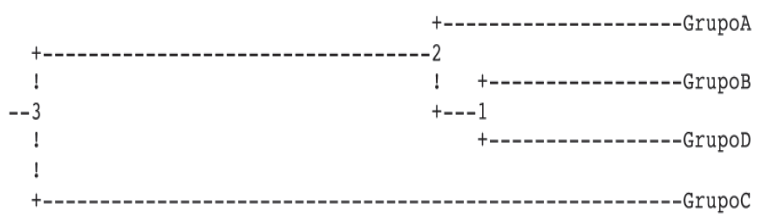

Figura 5. Dendrograma UPGMA de las distancias genéticas calculadas según Nei (GD) entre los grupos identificados en la colección de rosa de jamaica (A-D). Guatemala, mayo 2011.

Cuadro 3. Parámetros de diversidad genética para los grupos identificados en el análisis de agrupamientos de 17 genotipos de rosa de jamaica (A-D). Guatemala, mayo 2011.

\begin{tabular}{lcccccccc}
\hline Grupo & $\mathbf{N}$ & $\boldsymbol{P}$ & $\boldsymbol{\%}$ & $\mathbf{N e i}$ & $\mathbf{H}_{\mathbf{T}}$ & $\mathbf{H}_{\mathbf{s}}$ & $\mathbf{G}_{\text {ST }}$ & $\mathbf{N m}$ \\
\hline A & 3 & 46 & 38,66 & 0,1570 & & & & \\
B & 2 & 18 & 15,13 & 0,0627 & & & & \\
C & 1 & 00 & 00,00 & 0,0000 & & & & \\
D & 11 & 104 & 87,39 & 0,2856 & & & & \\
\hline Total & 17 & 117 & 98,32 & 0,3053 & 0,2980 & 0,1263 & 0,5762 & 0,3678 \\
\hline
\end{tabular}

Abreviaciones: Número de genotipos $(\mathrm{N})$, número de loci polimórficos $(P)$, porcentaje de loci polimórficos $(\%)$, diversidad genética según Nei (1978), diversidad genética total $\left(\mathrm{H}_{\mathrm{T}}\right)$, diversidad genética dentro de las poblaciones $\left(\mathrm{H}_{\mathrm{S}}\right)$, diferenciación genética $\left(\mathrm{G}_{\mathrm{ST}}\right)$ y flujo genético $(\mathrm{Nm})$. 
Cuadro 4. Identidad genética (I) y distancia genética de Nei (GD) calculadas según Nei (1978) para los cuatro grupos identificados dentro de los 17 genotipos de rosa de jamaica (A-D). Guatemala, mayo 2011.

\begin{tabular}{ccccc}
\hline GP & A & B & C & D \\
\hline A & ----- & 0,8350 & 0,6873 & 0,8529 \\
B & 0,1803 & ---- & 0,5944 & 0,8679 \\
C & 0,3750 & 0,5202 & ---- & 0,6477 \\
D & 0,1591 & 0,1417 & 0,4343 & ---- \\
\hline
\end{tabular}

Abreviaciones: Grupos (GP) identificados como A, B, C, D. Observación: Por encima de la diagonal se lee la identidad genética (I), por debajo de la diagonal se lee la distancia genética de Nei (GD).

Es probable que las características fenotípicas de precocidad y rendimiento superior observadas en dicho genotipo sean debidas a un fuerte componente genético. Un estudio de ligación de marcadores moleculares con estos descriptores de rendimiento puede identificar loci de características cuantitativas que asistan el mejoramiento genético de la rosa de Jamaica.

Entre los diecisiete genotipos de la colección hubo una alta diversidad genética natural. Además, no se encontraron genotipos duplicados a pesar de tener un número pequeño de individuos. Se observaron varias coincidencias entre los resultados de la caracterización agromorfológica y la molecular de los genotipos, pues se formaron conglomerados de accesiones con tiempos de cosecha y rendimientos de cáliz seco similares. Con estas observaciones se establece la posibilidad de una ligación cercana entre marcadores AFLPs y loci de características cuantitativas.

Se sugiere continuar saturando la matriz de AFLP con nuevas combinaciones selectivas a fin de identificar aquellas que ofrezcan más información y generen huellas digitales de ADN concluyentes. El mejoramiento genético de la rosa de jamaica en el futuro podría incluir estudios de ligación genética que permitan la selección asistida por marcadores de individuos con características de rendimiento superiores. A pesar de la variabilidad natural existente, generar una mayor diversidad por medio de un programa de cruzas o inducción de mutaciones puede ser de utilidad para mejorar algunas características de interés como resistencia a enfermedades y mayor contenido de ácidos hibiscus u otros componentes medicinales.

\section{LITERATURA CITADA}

Alcoces, N. 2009. Estudios citogenéticos de H. sabdariffa L. (Malveaceae). UDO Agrícola 9(3):595-598.

Blair, M; Diaz, J; Hidalgo, R; Diaz, L; Duque, M. 2007. Microsatellite characterization of Andean races of common bean (Phaseolus vulgaris L.). Theoretical Applied Genetics 116:29-43.

Chen, Z; Lu, B; Sameshima, K; Fu, D; Chen, J. 2004. Identification and genetic relationships of kenaf (Hibiscus cannabinus L.) germplasm revealed by AFLP analysis. Genetic Resources and Crop Evolution 51:393-401.

Dice, L. 1945. Measures of the amount of ecologic association between species. Ecology 26:297-302.

Gómez, J; Martínez, L; López, I; Silos, H; Ramírez, F; Andrade, I. 2008. Multiple shoot regeneration of Roselle (Hibiscus sabdariffa L.) from a shoot apix culture system. International Journal of Botany 4(3):326-330.

Hidalgo, S; De León, W; Ruano, H; Cano, L. 2009. Caracterización de trece genotipos de rosa de jamaica Hibiscus sabdariffa en Guatemala. Agronomía Mesoamericana 20(1):101-109.

Hussein, R; Shahein, Y; Hakim, A; Awad, H. 2010. Biochemical and molecular characterization of three colored types of roselle (Hibiscus sabdariffa L.). Journal of American Science 6(11):726-733.

Invitrogen Corporation. 2003. AFLP Analysis System I. Version B. User Manuel. USA. 18 p.

Jendereck, M; Shierenbeck, K; Olney, A. 1997. Development of randomly amplified polymorphisms DNA markers characteristic of Hibiscus rosa sinensis and H. syriacus. California Agricultural Technology Institute. Number 970902.7 p.

Nei, M. 1978. Estimation of average heterozygocity and genetic distance from a small number of individuals. Genetics 89:583-590.

Ponciano, K. 2004. Evaluación de técnicas de extracción de ADN y de visualización para marcadores microsatélites en caña de azúcar (Saccharum officinarum L.). Tesis de Licenciatura. UVG. Guatemala. 63 p.

Ponciano, K; Villatoro, J; Molina, L. 2009. Caracterización preliminar con microsatélites de la colección guatemalteca de frijol trepador. Agronomía Mesoamericana 20(2):245-254.

Rohfl, F.J. 1992. NTSYS-pc Numerical Taxonomy and Multivariate Analysis Systems. Versión 1.70. Exeter Publishing. New York, USA. 250p.

Rojas, P. 1999. Perspectivas de ampliación del Mercado de la jamaica (Hibiscus sabdariffa L.) del estado de 
Guerrero. Tesis de Licenciatura. Universidad Autónoma de Chapingo. División de Ciencias Económico Administrativas. México. 67 p.

Salvador, M; Seisdedos, M; Alia, R; Gil, L. 1997. Estudio de la variabilidad genética en doce poblaciones naturales de Pinus pinaster con marcadores isoenzimáticos. Cuadernos de la S.E.C.F. 5:119-124.

Solorzano, R; Macario, T. 2002. Estudio de la factibilidad del cultivo, procesamiento y comercialización de la rosa de jamaica. ALTERTEC/SEPAGRO. Guatemala. $16 \mathrm{p}$.

Tang, T; Zhong, Y; Jian, S; Shi, S. 2003. Genetic diversity of Hibiscus tiliaceus (Malvaceae) in China assessed using AFLP markers. Annals of Botany 92:409-414.
Torres, M; Escoto, M; Ron, J; Parra, G; Mena, S; Rodríguez, A; Rodríguez, A; Castellanos, O. 2011. Relationships among twelve genotypes of roselle (Hibiscus sabdariffa L.) cultivated in western Mexico. Industrial Crops and Products. Pendiente de publicación.

Wagara, I; Mwang'Ombe, A; Kimenju, J; Buruchara, R; Jamnadass, R; Majuwa, P. 2004. Genetic diversity of Phaeoisariopsis griseola in Kenya as revealed by AFLP and group-specific primers. Journal of Phytopathology 152:235-242.

Yeh, FY; Boyle, R; Ye, T; Mao, Z. 1997. POPGENE, the user friendly shareware for population genetic analysis. Versión 1.32. Molecular Biology and Biotechnology Centre, University of Alberta, Alberta, USA. 29 p. 